\title{
Optimal and Compromise Policies for Home Care: Schedul- ing Strategies of Local Government and Home Care Agency
}

\author{
Mari Ito *, Yosuke Nakamura *, Ryuta Takashima *
}

\begin{abstract}
We introduce a mixed integer programming model for home care schedules allocating home care workers to users' service time. The purpose of this scheduling is to reflect objectives of both a local government and a home care agency. The interest of the local government is to minimize the waiting times for home care users to avoid lowering their satisfaction levels. Meanwhile, the home care agency prefers home care workers to be able to visit as many care users' homes as possible each day. A formula is drawn out with the two interests in the objective function. In the numerical analysis, we use parameter values based on interviews conducted by the local government. Numerical results provide that the time window for home care service specified by home care users is the most important factor determining the home care schedule when the number of care workers available for the number of care users is sufficient. Conversely, when the number of workers is insufficient, it is the most crucial to consider the total transit time between the office of the home care agency and care users' homes. Furthermore, since it is the best strategy for the local government and the home care agency to select different schedules, it is important for them to reach a point of mutual compromise. For the society, we provide the important points to create a home care schedule with the two interests of the local government and home care agency.
\end{abstract}

Keywords: Home care, mixed integer programming, scheduling.

\section{Introduction}

All Japanese citizens aged 40 years and older purchase insurance in the care insurance system, and pay their care insurance premiums according to their income. Meanwhile, individuals certified with care need can receive care services according to their certification levels, i.e. 5 levels. A person requiring assistance for activities of daily living, such as housework and dressing, is in need of assistance, whereas a person requiring constant care due to bed-ridenness or dementia is in need of care. Every year, the number of individuals certified with care need is increasing as the population ages progressively [14]. With the increase in the number of individuals certified with care need, the amount of home care ser-

* Tokyo University of Science, Chiba, Japan 
vices increases, and the number of home care workers is rising with that as well. However, there is still a shortage of home care workers.

Home care workers denote individuals who provide care services that support the lives of care users in their homes. Tasks of home care services include elimination and feeding care, physical care, such as changing positions or bathing, and daily living support, such as preparing meals, laundry, shopping and cleaning users' living spaces. Examples of physical care include bathing assistance or drug administration. In 2013, about $42 \%$ of home care workers agreed that there was a shortage of human power [12]. The fact that there are certain home care services that home care workers can or cannot provide depending on their qualifications is another factor that worsens this sense of lack of human power.

In a survey about where people wished to die, the majority responded that they wished to die in their homes [13]. However, in 2014, the actual rate of people who died in their homes was $12.8 \%$. There seems to be a certain gap between the people's wishes and the reality. Home care users cannot live in their homes without home care services or family cooperation. Given the present-day situation in Japan with the increasing number of nuclear family households and households with both spouses working, anticipation for the role of home care workers is increasing.

The role of the local government is very important for the logistics of care services for the elderly [16]. The Japanese Ministry of Health, Labour and Welfare promotes to establish a "comprehensive community care system" by 2025 . This agenda aims to maintain people's lifestyles in their familiar communities for as long as possible until the end of their lives. Recently, comprehensive community support centers are being set up in cities, towns and villages as a core institution for improving public health care services for the elderly. The roles of them are creating care plans for home care users as home care service points.

The home care agencies serve a very significant role through supporting home care workers directly involved with at-home care. Home care schedules that allocate home care workers to users' service time are created by the home care agencies. Due to the increase in home care users and lack of human power, efficient visitation of care users' homes with the fewest number of home care workers is demanded in creating home care schedules that show when the home care workers visit the care users' homes in response. Furthermore, it must also satisfy many conditions, such as the qualifications held by home care workers, the times and services desired by home care users, and the available work hours of home care workers. Preparing home care schedules is extremely complicated.

A home care scheduling problem must be of social significance, given the review articles in recent years $[6,8,15]$. The problem is evaluated by means of mathematical optimization techniques since 20 years ago [1,5]. Authors use different constraints and objectives because home care management differs from country to country [2, 4, 7, 9, 10, 17]. As studies similar to Japanese home care management, Mankowska et al. [11] represented delay time in arriving at the care users' homes as the penalty constant in a case where home care services are distinguished by means of the number as single and double ones. They proposed a mathematical model to minimize the sum of transit time of all home care workers. They also performed a numerical analysis with a scenario comprising 4 home care services, 3 home care workers, and 7 home care users. Braekers et al. [3] proposed a mathematical model and a metaheuristic solution approach to minimize the total cost of transit and maximize the home care user satisfaction. They could obtain multiple schedules and clarify the relationship of tradeoff relation between the total cost and the home care user inconvenience. Constraints of Braekers et al. [3] differ that of Japanese home care management, but we propose objective function based on their study. The previous studies did 
not determine optimal schedules from the two points of the local government that manages home care users and the home care agency that manages home care workers. Considering the social background in which the role of the local government for care for the elderly is highly valued, it is important to manage home care services from both the standpoints of the government that supports home care users and the home care agency that manages home care workers.

In this study, we propose a mixed integer programming model with an objective function that can be weighted for the interests of the local government and the home care agency. The interest of the local government is to minimize the waiting time for home care users, that is preventing their satisfaction from falling. Meanwhile, the home care agency's interest is to maximize the number of care users' homes that each home care worker can visit in a day. In general, the home care schedules are created by the home care agencies; thus, most schedules tend to favor the interests of the home care agency. Taking both standpoints into consideration will enable scheduling that ensures home care users' satisfaction. We evaluate the effects of the difference in the priority of the interests of the local government and the home care agency on optimal home care schedules through numerical analysis. Additionally, we investigate whether there is an option that is optimal for both parties, or if a point of compromise must be found. The used data is generated based on the interviews that the Nagareyama city hall conducted as a home care management. From numerical analyses, we provide the following four important points to create a home care schedule:

- The home care agencies recommend home care users to request time windows for service that offer some leeway.

- The home care agencies determine the start time of services while considering the home care workers' current task schedules.

- The home care agencies create home care schedules by considering a table of transit time between the agency and/or users' homes.

- The local government and the home care agency mutually agree upon a point of compromise.

The results imply that each local government could make a decision for home care in order to meet the objectives and resources of each home care agency.

This paper models the local government and home care agency perspectives and describes them in terms of optimization research. For the society, we provide the four important points to create a home care schedule with the two interests of the local government and home care agency.

The remainder of this paper is organized as follows: Section 2 introduces the proposed model. Section 3 presents a numerical analysis of the proposed model. Section 4 presents our concluding remarks along with suggestions for future research.

\section{Home Care Scheduling}

\subsection{Outline}

In this study, home care schedules that meet the demands of both the home care agency and local government are created. Care workers are managed by home care offices, which provide multiple home care services. Home care services may be single or double services. 
Single services are provided by one care worker at one service operation. Double services are provided by two care workers, and comprise two service operations. Double services further divide into two types: the first consisting of services that require "priority," i.e., being completed in advance, such as waiting for a period of time after administering a drug that must be taken before a meal, followed by the second service of meal preparation and serving; and the second type consisting of services that can be provided simultaneously by two care workers. Here, care workers first meet at the home care office, are routed to care users' homes, then complete their day at the home care office. In the objective function of the model proposed herein, care workers' total transit time and delays in arriving at the care users' homes (waiting time for the home care user) have been weighed to minimize their sum.

\subsection{Model}

We formulate the problem as the mixed integer programming problem. The formulation is shown as follows:

Notation

Indices.

$s_{1}$ : first service to be provided in double service.

$s_{2}$ : second service to be provided in double service.

$w_{1}$ : home care worker that provides $s_{1}$.

$w_{2}$ : home care worker that provides $s_{2}$.

Index sets.

$U$ : the set of care users' homes.

$S:$ the set of home care services.

$W$ : the set of home care workers.

$U^{0}$ : the set of care users' homes and home care agency, $U^{0}=U \cup\{0\}$.

$U^{s}$ : the set of care users' homes of single service.

$U^{d}$ : the set of care users' homes of double services, $U^{d}=U^{\text {sim }} \cup U^{\text {prec }}$.

$U^{s i m}$ : the set of care users' homes requiring simultaneous services.

$U^{p r i}$ : the set of care users' homes requiring prioritization services.

Parameters.

$a_{w s}: 1$ if home care worker $w$ is qualified to provide home care service $s$, and 0 otherwise, $(w \in W, s \in S)$.

$r_{u s}: 1$ if care user's home $u$ requires home care service $s$, and 0 otherwise, $(u \in U, s \in S)$.

$\delta_{u}^{\text {min }}:$ minimum time interval between the start time of two home care services at care user's home $u$, where $\delta_{u}^{\min }=0$ when $u \in U^{\text {sim }},\left(u \in U^{d}\right)$. 
$\delta_{u}^{\max }:$ maximum time interval between the start time of two home care services at care user's home $u$, where $\delta_{u}^{\max }=0$ when $u \in U^{\text {sim }},\left(u \in U^{d}\right)$.

$\left[e_{u}, l_{u}\right]$ : time window for home care service at care user's home $u, e_{u}$ : start time of desired home care service at care user's home $u, l_{u}$ : end time of desired home care service at care user's home $u,(u \in U)$.

$d_{u k}$ : transit time from one home care agency or care user's home $u$ to another user's home $k,\left(u \in U^{0}, k \in U^{0}\right)$.

$p_{u s}$ : time spent on carrying out home care service $s$ at care user's home $u,(u \in U, s \in S)$.

$b$ : maximum number of care users' homes that a home care worker can visit in a day.

$\alpha$ : weighting factor, i.e. exogenous policy parameter that depends on a relationship between the local government and the home care agency, $\alpha \in[0,1]$.

M: large number.

Variables.

$x_{u k w s}: 1$ if home care worker $w$ transits from one care user's home $u$ to another $k$ to carry out home care service $s$, and 0 otherwise, $\left(u \in U^{0}, k \in U^{0}, w \in W, s \in S\right)$.

$T^{\max }$ : maximum delay time in arriving at a care user's home.

$T$ : total delay time in arriving at all care users' homes.

$D$ : sum of transit time of all home care workers.

$t_{u w s}: \quad$ start time for home care worker $w$ to provide home care service $s$ at care user's home $u,(u \in U, w \in W, s \in S)$.

$z_{u s}$ : delay from scheduled time of completion of home care service $s$ per care user's home $u,(u \in U, s \in S)$.

\section{Formulation}

$$
\text { Minimize } \quad \alpha D+(1-\alpha)\left(T+T^{\max }\right)
$$

subject to

$$
\begin{gathered}
D=\sum_{w \in W} \sum_{u, k \in U^{0}} \sum_{s \in S} d_{u k} x_{u k w s}, \\
T \geq \sum_{u \in U} \sum_{s \in S} z_{u s}, \\
T^{m a x} \geq z_{u s}, \quad \forall u \in U, \forall s \in S, \\
\sum_{u \in U^{0}} \sum_{s \in S} x_{0 u w s}=\sum_{u \in U^{0}} \sum_{s \in S} x_{u 0 w s}=1, \quad \forall w \in W,
\end{gathered}
$$




$$
\begin{gathered}
\sum_{k \in U^{0}} \sum_{s \in S} x_{k u w s}=\sum_{k \in U^{0}} \sum_{s \in S} x_{u k w s}, \quad \forall u \in U, \forall w \in W \\
\sum_{w \in W} \sum_{k \in U^{0}} a_{w s} x_{k u w s}=r_{u s}, \quad \forall u \in U, \forall s \in S \\
t_{u w s_{1}}+p_{u s_{1}}+d_{u k} \leq t_{k w s_{2}}+M\left(1-x_{u k w s_{2}}\right), \quad \forall u \in U^{0}, \forall k \in U, \forall w \in W, \forall s_{1}, s_{2} \in S, \\
t_{u w s} \geq e_{u}, \quad \forall u \in U, \forall w \in W, \forall s \in S, \\
t_{u w s} \leq l_{u}+z_{u s}, \quad \forall u \in U, \forall w \in W, \forall s \in S \\
t_{u w_{2} s_{2}}-t_{u w_{1} s_{1}} \geq \delta_{u}^{m i n}-M\left(2-\sum_{k \in U^{0}} x_{k u w_{1} s_{1}}-\sum_{k \in U^{0}} x_{k u w_{2} s_{2}}\right) \\
\forall u \in U^{d}, \forall w_{1}, w_{2} \in W, \forall s_{1}, s_{2} \in S: s_{1}<s_{2}, \\
t_{u w_{2} s_{2}}-t_{u w_{1} s_{1}} \leq \delta_{u}^{m a x}+M\left(2-\sum_{k \in U^{0}} x_{k u w_{1} s_{1}}-\sum_{k \in U^{0}} x_{k u w_{2} s_{2}}\right) \\
\forall u \in U^{d}, \forall w_{1}, w_{2} \in W, \forall s_{1}, s_{2} \in S: s_{1}<s_{2}, \\
x_{u k w s} \in\left\{0, a_{w s} r_{k s}\right\}, \quad \forall u, k \in U^{0}, \forall w \in W, \forall s \in S \\
t_{u w s}, z_{u s} \geq 0, \quad \forall u \in U^{0}, \forall w \in W, \forall s \in S \\
\sum_{s \in S_{u}, k \in U^{0}} \sum_{u k w s} \leq b+1, \quad \forall w \in W .
\end{gathered}
$$

In the above formulation, objective function (1) is comprised of two terms with weight parameters $\alpha$ and $1-\alpha$. The first term of the objective function minimizes the sum of transit time of all care workers. The second term of the objective function minimizes delay time in arriving at the users' homes, i.e., waiting time for the home care user. For example, when $\alpha=1$, this problem is just to minimize the sum of transit time of all care workers $D$. This implies that the policy is implemented just by decisions of the home care agency. Conversely, when $\alpha=0$, this problem is just to minimize delays in arriving at the care users' homes, i.e., waiting time for the home care user. This implies that the policy is implemented just by the decisions of the local government.

Constraint (2) defines the sum of the home care workers' transit time, $D$. Constraint (3) defines total delay time in arriving at all care users' homes, $T$. Constraint (4) defines maximum delay time in arriving at a care user's home, $T^{\max }$. Constraint (5) sets the start and the end of the care workers' route as the home care agency. Constraint (6) describes the conditions for home care workers' inflow and outflow. Constraint (7) assigns qualified home care workers to care users depending on a kind of care services, $r_{u s}$. Constraint (8) determines the service start time. By constraint (9), home care services are carried out after the desired start time, $e_{u}$. By constraint (10), home care services are carried out within the time that includes delays. Constraint (11) shows the minimum interval between service start times. Constraint (12) shows the maximum interval between service start times. Constraint (13) defines the range for $x_{u k w s}$ values. Constraint (14) is the non-negativity constraint. Constraint (15) is the upper limit for the number of care users' homes that a care worker can visit in one day. 
Table 1: Start and end time of desired home care services at a user's home, $\left[e_{u}, l_{u}\right]$.

\begin{tabular}{lll} 
& Nine-home care scheduling & Sixteen-home care scheduling \\
\hline Care user's home 1 & {$[4 \mathrm{pm}, 5 \mathrm{pm}]$} & {$[12 \mathrm{am}, 13 \mathrm{pm}]$} \\
Care user's home 2 & {$[3 \mathrm{pm}, 4 \mathrm{pm}]$} & {$[12 \mathrm{am}, 13 \mathrm{pm}]$} \\
Care user's home 3 & {$[1 \mathrm{pm}, 2 \mathrm{pm}]$} & {$[9 \mathrm{am}, 10 \mathrm{am}]$} \\
Care user's home 4 & {$[4 \mathrm{pm}, 5 \mathrm{pm}]$} & {$[11 \mathrm{am}, 12 \mathrm{am}]$} \\
Care user's home 5 & {$[2 \mathrm{pm}, 3 \mathrm{pm}]$} & {$[14 \mathrm{pm}, 15 \mathrm{pm}]$} \\
Care user's home 6 & {$[1 \mathrm{pm}, 2 \mathrm{pm}]$} & {$[15 \mathrm{pm}, 16 \mathrm{pm}]$} \\
Care user's home 7 & {$[1 \mathrm{pm}, 2 \mathrm{pm}]$} & {$[11 \mathrm{am}, 12 \mathrm{am}]$} \\
Care user's home 8 & {$[4 \mathrm{pm}, 5 \mathrm{pm}]$} & {$[11 \mathrm{am}, 12 \mathrm{am}]$} \\
Care user's home 9 & {$[9 \mathrm{am}, 10 \mathrm{am}]$} & {$[13 \mathrm{pm}, 14 \mathrm{pm}]$} \\
Care user's home 10 & - & {$[17 \mathrm{pm}, 18 \mathrm{pm}]$} \\
Care user's home 11 & - & {$[14 \mathrm{pm}, 15 \mathrm{pm}]$} \\
Care user's home 12 & - & {$[11 \mathrm{am}, 12 \mathrm{am}]$} \\
Care user's home 13 & - & {$[9 \mathrm{am}, 10 \mathrm{am}]$} \\
Care user's home 14 & - & {$[10 \mathrm{am}, 11 \mathrm{am}]$} \\
Care user's home 15 & - & {$[13 \mathrm{pm}, 14 \mathrm{pm}]$} \\
Care user's home 16 & - & {$[11 \mathrm{am}, 12 \mathrm{am}]$} \\
\hline
\end{tabular}

\section{Numerical Experiments}

\subsection{Data}

We set the parameters for mathematical analysis based on surveys conducted by the $\mathrm{Na}$ gareyama city hall via home care agencies. A target period of 1 day with increments of 1 min is studied. Numerical analysis is performed for the following two scenarios. Herein, we analyze the influence of the weighting factor of the objective function on the schedule by solving nine-home care scheduling problem. Furthermore, in order to examine the influence of increasing the number of care users on the schedule, the sixteen-home care scheduling problem is solved. This is maximum number of care users for all 3 care workers to be within the upper limit for the number of visiting houses; 16 care users' homes. We consider nine and sixteen-home care scheduling problems with 4 home care services, i.e., $U=9$ or 16 , and $S=4$. Home care workers $W$ are given 3 people with service qualifications. Home care worker 1 can provide home care services 1,2 , and 4 . Home care worker 2 can provide home care services 3 and 4 . Home care worker 3 can provide all home care services. They are related to the setting of home care workers' qualifications, $a_{w s}$. Since the number of home care users who receive double care services only represent a small fraction, simultaneous services $U^{\text {sim }}$ and prioritized services $U^{\text {pri }}$ are each set for care user's home 8 and care user's home 9 , i.e., $U^{s}$ is care users' homes $1-7$. $U^{d}$ is care users' homes 8 and 9 with nine-home care scheduling problem. As sixteen-home care scheduling problem, simultaneous services $U^{\text {sim }}$ and prioritized services $U^{p r i}$ are each set for care user's home 15 and care user's home 16 , i.e., $U^{s}$ is care users' homes $1-14$. $U^{d}$ is care users' homes 15 and 16.

Care user's homes 8 and 15 do not have the time interval between the start time of two home care services because of simultaneous services $U^{\text {sim }}$, i.e., $\delta_{8}^{\min }$ and $\delta_{8}^{\max }=0$, and $\delta_{15}^{\min }$ and $\delta_{15}^{\max }=0$. In contrast, care user's home 9 and 16 have a minimum and a maximum time interval between the start time of two home care services because of prioritized services $U^{\text {pri }}$, i.e., $\delta_{9}^{\min }$ and $\delta_{16}^{\min }=10$, and $\delta_{9}^{\max }$ and $\delta_{16}^{\max }=30$. Home care services required 


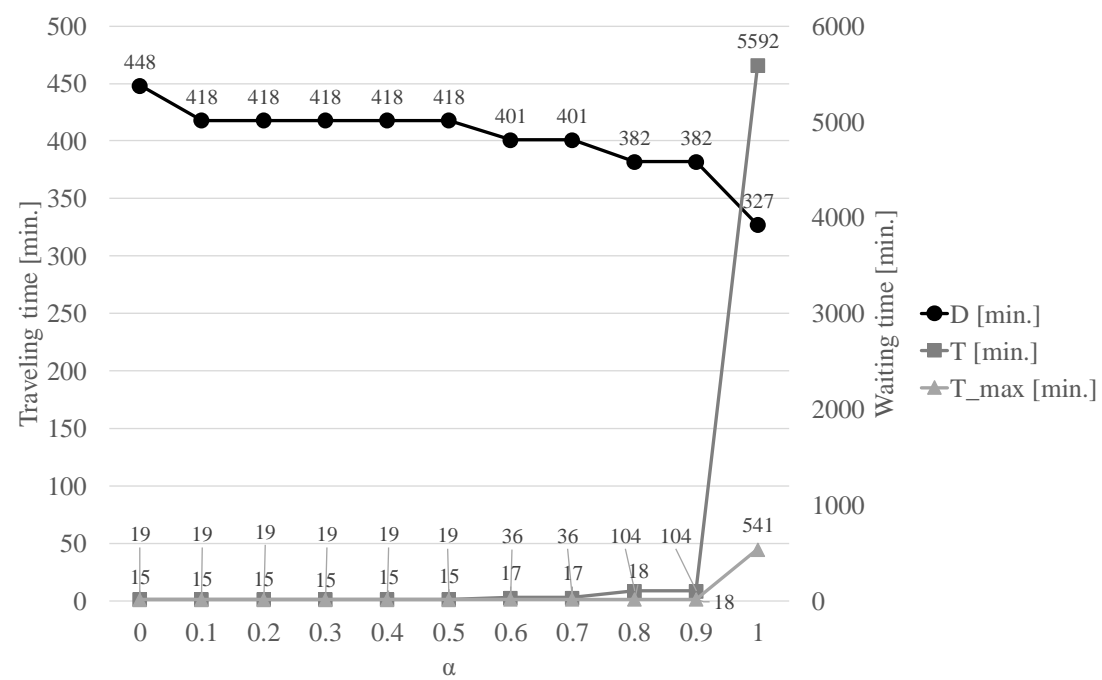

Figure 1: Transit and waiting time of nine-home care scheduling problem under different $\alpha$ [min.].

by care users are shown in Table 3. This is related to the setting of $r_{u s}$.

The start and end time of desired home care services at a care user's home $\left[e_{u}, l_{u}\right]$ are shown in Table 1. The time window for a care user's home $\left[e_{u}, l_{u}\right]$ are set in 1-hr units between 9 am to $6 \mathrm{pm}$. Transit time from one home agency or care user's home to another user's home are shown in Table 2. The transit time $d_{u k}$ are set in 1-min units between $20 \mathrm{~min}$ to $40 \mathrm{~min}$. Time spent on carrying out a home care service at a care user's home are $p_{u 1}=45, p_{u 2}=50, p_{u 3}=50, p_{u 4}=55$ with nine-home care scheduling problem. As sixteen-home care scheduling problem, time spent on carrying out a home care service at a care user's home are $p_{u 1}=40, p_{u 2}=30, p_{u 3}=30, p_{u 4}=40$. The service time $p_{u s}$ are set in 5-min units between $30 \mathrm{~min}$ to $55 \mathrm{~min}$. $\left[e_{u}, l_{u}\right], d_{u k}$, and $p_{u s}$ are created uniformly by generating random integers. The maximum number of homes that a care worker can visit in a day $b$ is 6 homes. The large number $M$ is 500 .

\subsection{Results of nine-home care scheduling}

We provide some numerical examples for various weighting factors of $\alpha \in[0,1]$ to assess the effects of the weighting factor $\alpha$ on the optimal schedule. The computational environment was an Intel(R) Core(TM) i5-6200U CPU @ 2.30 GHz with 8.00 GB RAM. The CPU time requested to solve the nine-home care scheduling problem was approximately $50 \mathrm{sec}$ a solution on an average using the IBM ILOG CPLEX Optimization Studio version 12.6.3 solver. In the generated test instances, there were 5,018 constraints and 1,359 variables.

Figure 1 shows the transit and waiting time of nine-home care scheduling problem under different $\alpha$. The case of $\alpha=0$ minimizes the home care workers' delay time, i.e., waiting time for home care user, $T+T^{\max }$. Conversely, the case of $\alpha=1$ minimizes the sum of transit time of all home care workers, $D$. By Figure 1, the transit time $D$ decreased relatively slowly as the value of an increased. There was almost no change in maximum care workers' delay time $T^{\max }$ between $\alpha=0$ and $\alpha=0.9$, but increased dramatically at $\alpha=1$. Likewise, there was almost no change in the sum of care workers' delay time $T$ for $\alpha=0$ to $\alpha=0.9$, but increased when $\alpha=1$; that is, it shows that the waiting time for home 


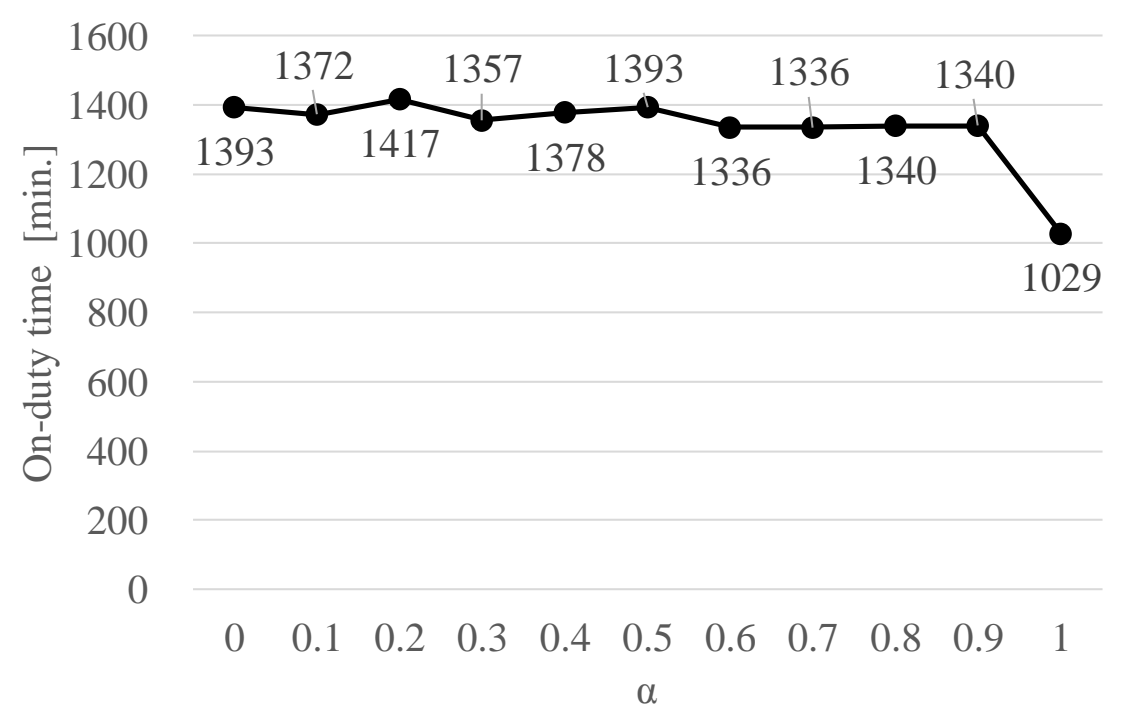

Figure 2: Work hours of all home care workers of nine-home care scheduling problem under different $\alpha$ [min.].

care users increased drastically.

Figure 2 shows the work hours of all home care workers of 9 home care scheduling problem under different $\alpha$. Work hours represent the time from the departure of all home care workers from the home care agency to their return at the home care agency. From Figure 2, the work hours of all home care workers decreased markedly when $\alpha=1$. This was because the number of dispatched home care workers could be reduced from 3 to 2 by accounting for home care workers' delay time at $\alpha=1$ to minimize their transit time.

Table 4 shows the nine-home care schedules under different $\alpha$. Table 4 shows the schedule in which home care worker 1 visited 3 care users' homes, and home care worker 3 visited 5 care users' homes, at $\alpha \leq 0.5$. However, when $0.6 \leq \alpha \leq 0.9$, schedules in which home care worker 1 visited 4 care users' homes in the order of $\langle 9,6,7,8\rangle$ and home care worker 3 visited 4 care users' homes were created. Home care worker 2 visited 3 care users' homes in the order of $\langle 3,2,8\rangle$ in all cases at $\alpha \leq 0.9$. Furthermore, when $\alpha=1$, home care worker 1 visited 5 homes, and home care worker 3 visited 6 homes; two workers have performed all the services.

By Figures 1 and 2, schedules that minimize transit time of home care worker is recommended if the home care agency aims to cut labor costs. By Figure 1, the local government has minimum waiting time $T+T^{\max }$ when $0 \leq \alpha \leq 0.5$. In contrast, the agency has minimum transit time $D$ when $\alpha=1$, but this would dramatically prolong waiting time $T+T^{\max }$. That is, the local government has maximum $T+T^{\max }$ when $\alpha=1$. In contrast, the home care agency has maximum transit time $D$ when $\alpha=0$. Therefore, the compromised schedule between the local government and the home care agency is at $0.1 \leq \alpha \leq 0.9$. This means that decision-making for the agency is relatively prioritized over that for local government. This result is consistent with a policy of Nagareyama city in which home care service is practically outsourced to the home care agency. On the other hand, in other cities, the local governments partially might decide the schedule and the resource allocation for home care service. In the case, a policy direction and preferred objective for home care service, that is, the policy parameter $\alpha$ would be derived through a negotiation. 


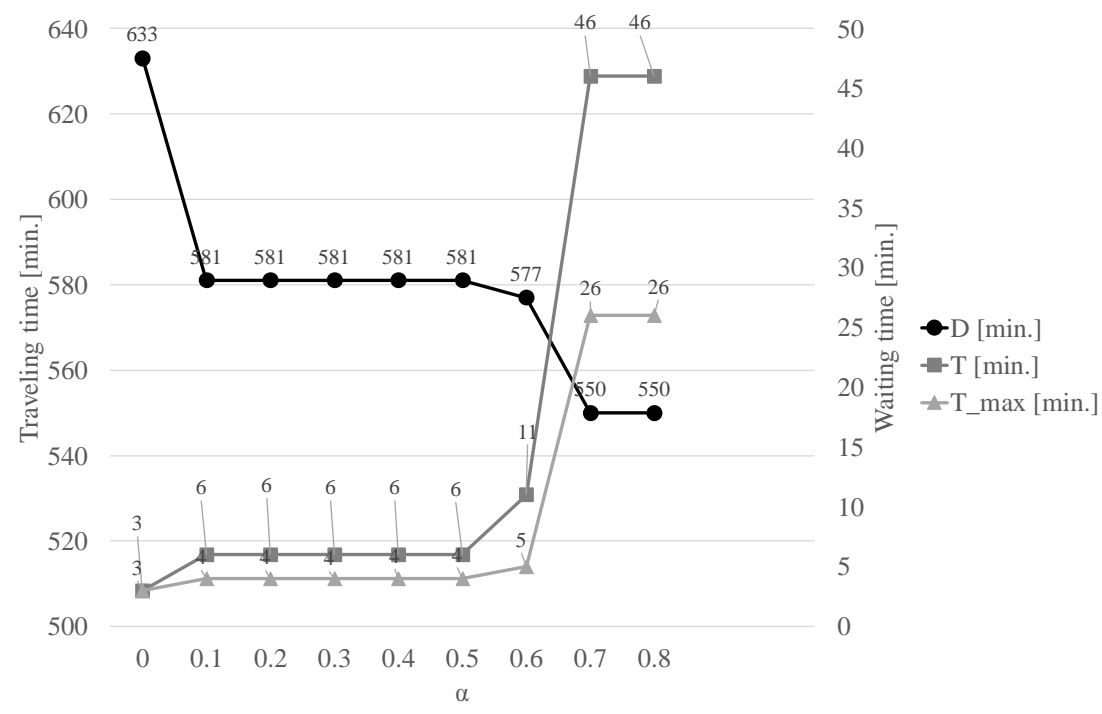

Figure 3: Results of sixteen-home care scheduling problem under different $\alpha$ [min.].

Combining Tables 1 and 4 suggests that the schedule on Table 4 shows that settings for the time windows of home care users on Table 1 largely impact the order visiting care users' homes and the start time of services. That is, settings for the care users' time windows is the most important factor in arranging home care schedules. However, the current task schedules of the home care workers are not considered in the request of time windows for home care service. We recommend asking home care users to request time windows with some leeway to allow for a compromise between agencies' and home care users' schedules while considering the current task schedules of the home care workers. The preferred home care schedule provides them to enough time in which the home care agency manages and operates the home care users and the home care workers.

\subsection{Results of sixteen-home care scheduling}

We solve sixteen-home care scheduling problem to analyze the influence of increasing the number of users on the schedule. In a social background where the number of workers is expected to be insufficient in the future, we investigate the optimal schedule when we assume that all 3 workers visit the largest number of users' homes a day. In the generated test instances, there were 14,111 constraints and 3,739 variables. When $\alpha=0.9$ and 1 , the solution could not be found due to insufficient memory. In Table 5, we show the CPU times of sixteen-home care scheduling corresponding to each value of $\alpha$.

Figure 3 shows the results of sixteen-home care scheduling under different $\alpha$. The sum of all care workers' transit time $D$ decreased dramatically between $\alpha=0$ and 0.1 . The maximum delay time of care workers $T^{\max }$ increased suddenly between $\alpha=0.6$ and 0.7 . The total delay time of care workers $T$ also increased between $\alpha=0.6$ and 0.7. Figure 4 shows the objective function values of sixteen-home care scheduling under different $\alpha$. In Figure 4, When $\alpha=0$, the value of the objective function was lowest. In contrast, the value of the objective function was highest when $\alpha=0.8$. That is, schedules that minimize both care workers' transit time $D$ and care workers' delay times $T+T^{\max }$ were optimal. Figure 5 shows the actual hours of all care workers of sixteen-home care scheduling problem under 


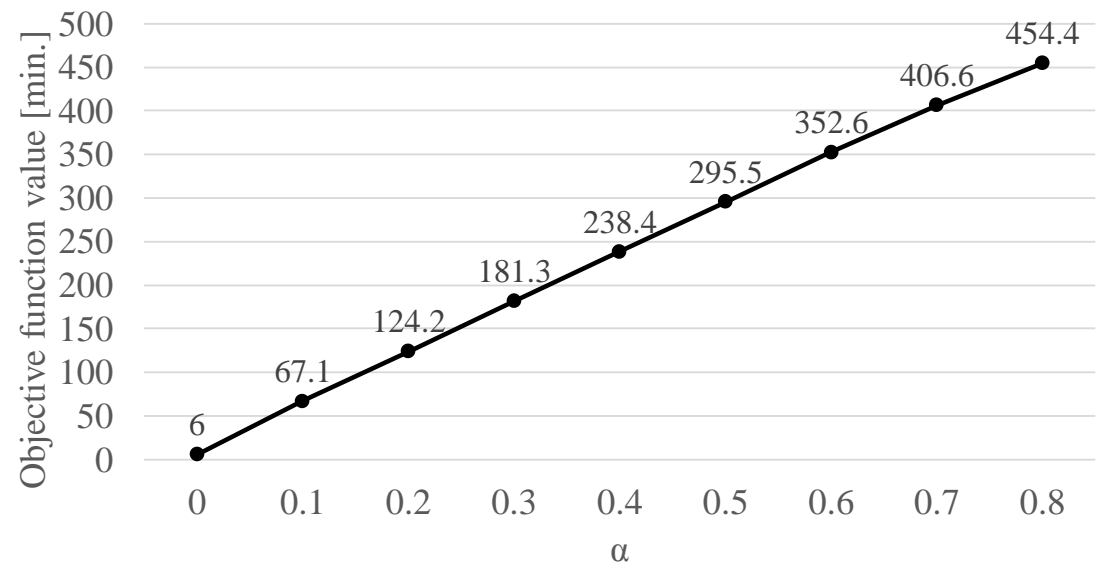

Figure 4: Objective function values of sixteen-home care scheduling problem under different $\alpha$ [min.].

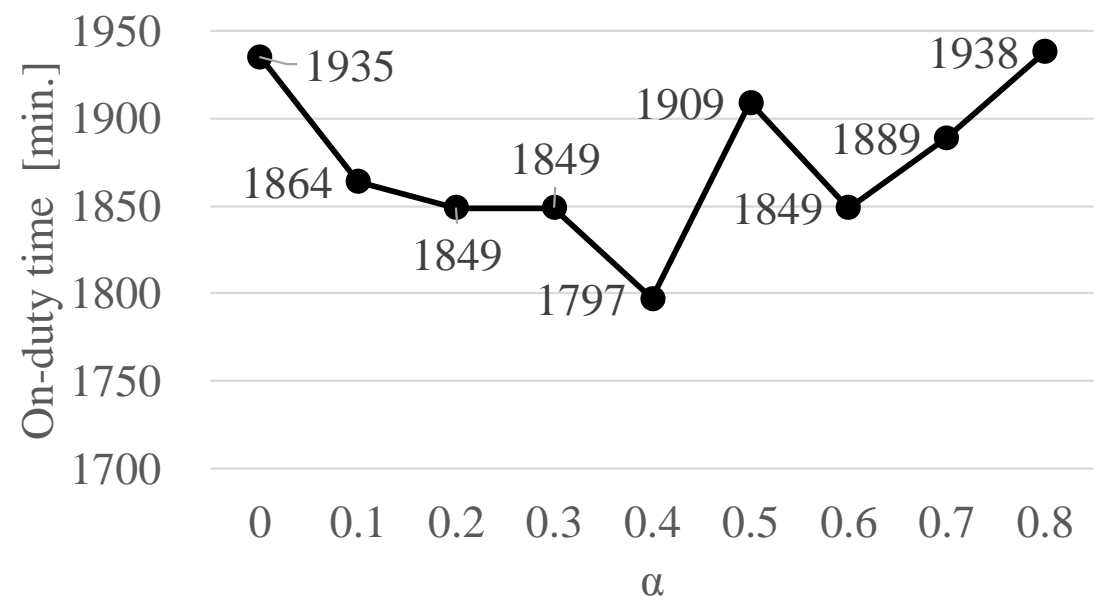

Figure 5: Actual hours of all care workers of sixteen-home care scheduling problem under different $\alpha$ [min.].

different $\alpha$. Figure 5 shows that actual hours at work of all care workers decreased dramatically when $\alpha=0.4$. This is influenced by the fact that care worker 2's actual hours at work decreased. When the number of care users is equal to the maximum number of care users that a care worker can visit in a day, care workers take the maximum number of rotations for any value; therefore, there were no major changes to the number of actual hours at work. Table 6 shows the sixteen-home care schedules under different $\alpha$. Table 6 shows a schedule that shows that all care workers visit a total of 6 homes in one day. When $\alpha=0$, a schedule that was not in accordance with the time windows for the care users as shown in Table 3 was created. This shows that the schedule according to the time windows is not feasible when all care workers' delay times are minimized. The schedule created when considering weighting factors at $0.1 \leq \alpha$ gave almost the same results. This is determined by a service pair with shorter transit time than the transit time between the home care office and/or between care users' homes shown in Table 2. For example, in a scenario with $\alpha=0.7$ and 0.8, i.e., 
a schedule in which the schedule is created with more weight placed on transit time than delay time of care workers, the resulting schedule indicated visiting the home of care user 9 before care user 10's, which minimizes care worker 1's transit time between care users' homes. We recommend home care agencies to create care workers' schedules after creating a table similar to Table 2 that lists transit times between home care office and/or care users' homes. This scenario assumes the maximum number of care users for the number of care workers, thus the time windows of care users did not affect the schedules of care workers.

In the present numerical analysis, the schedule compromising the municipal office and home care office was at $0.1 \leq \alpha \leq 0.5$ or $\alpha=0.6$. From the standpoint of the municipal office, $T+T^{\max }$ is lowest when $\alpha=0$. However, the value for transit time is highest when $\alpha=0$ compared to others. A glance through $0.1 \leq \alpha \leq 0.5$ shows that the value of $T+T^{\max }$ increases somewhat, suggesting that the schedule when $0.1 \leq \alpha \leq 0.5$ should be selected. The schedule with $\alpha=0.7$ or 0.8 would be desired with the focus on $D$ from the standpoint of the home care office; however, this would lengthen users' waiting times exceedingly. Thus, it would be desirable to select the schedule at $\alpha=0.6$, which has the next smallest value of $D$.

\section{Conclusion}

We developed the mixed integer programming model with an objective function that can be weighted for the interests of the local government and the home care agency. We investigated the effects of interests of the local government and the home care agency on scheduling. Used data was generated based on interviews that the Nagareyama city hall, Chiba prefecture conducted on home care agencies. The results showed that the settings for the time windows for home care services that home care users requested were the most important factor determining the schedule when the number of home care workers was sufficient for the number of home care users. Thus, we recommend home care agencies to request home care users to request time windows that offer some leeway while considering the home care workers' current task schedules to reach a compromise between home care agencies and home care users. Furthermore, we found care worker schedules could not satisfy the time windows requested by the users when the number of care workers was insufficient for the number of care users. Therefore, we recommend home care providers to create care workers' schedules only after creating a table of transit time between the home care office and/or care users' homes. Moreover, the optimal strategy for the local government and the home care agency would be to select different schedules, demonstrating the importance of finding a mutually-agreed point of compromise.

In the future, the proposed model should be expanded to a model considering the home care workers' workload, identifying the home care worker that home care users wish to appoint and incorporating cost calculations.

\section{Appendixes}

\section{Upper bound of large number $M$}

Formulation (11) is developed as follows:

$$
t_{i v_{2} s_{2}}-t_{i v_{1} s_{1}} \geq \delta_{i}^{\min }-M\left(2-\sum_{j \in C^{0}} x_{j i v_{1} s_{1}}-\sum_{j \in C^{0}} x_{j i v_{2} s_{2}}\right),
$$




$$
\begin{gathered}
\delta_{i}^{m i n}-M\left(2-\sum_{j \in C^{0}} x_{j i v_{1} s_{1}}-\sum_{j \in C^{0}} x_{j i v_{2} s_{2}}\right) \leq t_{i v_{2} s_{2}}-t_{i v_{1} s_{1}}, \\
-M\left(2-\sum_{j \in C^{0}} x_{j i v_{1} s_{1}}-\sum_{j \in C^{0}} x_{j i v_{2} s_{2}}\right) \leq t_{i v_{2} s_{2}}-t_{i v_{1} s_{1}}-\delta_{i}^{m i n}, \\
M \geq \frac{-t_{i v_{2} s_{2}}+t_{i v_{1} s_{1}}+\delta_{i}^{\text {min }}}{\left(2-\sum_{j \in C^{0}} x_{j i v_{1} s_{1}}-\sum_{j \in C^{0}} x_{j i v_{2} s_{2}}\right)} .
\end{gathered}
$$

Formulation (12) is developed as follows:

$$
\begin{gathered}
t_{i v_{2} s_{2}}-t_{i v_{1} s_{1}} \leq \delta_{i}^{m a x}+M\left(2-\sum_{j \in C^{0}} x_{j i v_{1} s_{1}}-\sum_{j \in C^{0}} x_{j i v_{2} s_{2}}\right), \\
\delta_{i}^{m a x}+M\left(2-\sum_{j \in C^{0}} x_{j i v_{1} s_{1}}-\sum_{j \in C^{0}} x_{j i v_{2} s_{2}}\right) \geq t_{i v_{2} s_{2}}-t_{i v_{1} s_{1}}, \\
M\left(2-\sum_{j \in C^{0}} x_{j i v_{1} s_{1}}-\sum_{j \in C^{0}} x_{j i v_{2} s_{2}}\right) \geq t_{i v_{2} s_{2}}-t_{i v_{1} s_{1}}-\delta_{i}^{\max }, \\
M \geq \frac{t_{i v_{2} s_{2}}-t_{i v_{1} s_{1}}-\delta_{i}^{\max }}{\left(2-\sum_{j \in C^{0}} x_{j i v_{1} s_{1}}-\sum_{j \in C^{0}} x_{j i v_{2} s_{2}}\right)} .
\end{gathered}
$$

By using formulation (8),

$$
\begin{gathered}
t_{i v s_{1}}+p_{i s_{1}}+d_{i j} \leq t_{j v s_{2}}+M\left(1-x_{i j v s_{2}}\right), \\
M\left(1-x_{i j v s_{2}}\right) \geq t_{i v s_{1}}+p_{i s_{1}}+d_{i j}-t_{j v s_{2}}, \\
M \geq \frac{t_{i v s_{1}}+p_{i s_{1}}+d_{i j}-t_{j v s_{2}}}{1-x_{i j v s_{2}}}, \\
-t_{i v_{2} s_{2}}+t_{i v_{1} s_{1}}+\delta_{i}^{\min } \leq t_{i v_{2} s_{2}}-t_{i v_{1} s_{1}}-\delta_{i}^{\max }, \\
\delta_{i}^{\max }+\delta_{i}^{\min } \leq 2\left(t_{i v_{2} s_{2}}-t_{i v_{1} s_{1}}\right), \\
\frac{t_{i v_{2} s_{2}}-t_{i v_{1} s_{1}}-\delta_{i}^{\max }}{\left(2-\sum_{j \in C^{0}} x_{j i v_{1} s_{1}}-\sum_{j \in C^{0}} x_{j i v_{2} s_{2}}\right)} \geq \frac{t_{i v s_{1}}+p_{i s_{1}}+d_{i j}-t_{j v s_{2}}}{1-x_{i j v s_{2}}} .
\end{gathered}
$$

i.e., the upper bound of the rough is

$$
M \geq \frac{t_{i v_{2} s_{2}}-t_{i v_{1} s_{1}}-\delta_{i}^{\max }}{\left(2-\sum_{j \in C^{0}} x_{j i v_{1} s_{1}}-\sum_{j \in C^{0}} x_{j i v_{2} s_{2}}\right)} .
$$




\section{Acknowledgments}

The authors would like to thank staff of Nagareyama city hall in Chiba prefecture and the agencies in this city for valuable comments. This work is partially supported by JSPS KAKENHI (Japan) Grant Number JP 21K14371.

\section{References}

[1] S.V. Begur, D.M. Miller, and J.R. Weaver, "An Integrated Spatial DSS for Scheduling and Routing Home Healthcare Nurses," Interfaces, vol. 27, 1997, pp. 35-48.

[2] V. Borsani, A. Matta, G. Beschi, and F. Sommaruga, "A Home Care Scheduling Model for Human Resources," Proceedings of the International Conference on Service Systems and Service Management, vol. 1, 2006, pp. 449454.

[3] K. Braekers, R.F. Hartl, S.N. Parragh, and F. Tricoire, "A Bi-objective Home Care Scheduling Problem: Analyzing the Trade-off Between Costs and Client Inconvenience,” European Journal of Operational Research, vol. 248, 2016, pp. 428-443.

[4] P. Cappanera, M.G. Scutell, F. Nervi, and L. Galli, "Demand Uncertainty in Robust Home Care Optimization," Omega, vol. 80, 2017, pp. 95-110.

[5] E. Cheng, and J.L. Rich, "A Home Health Care Routing and Scheduling Problem," Technical report CAAM TR98-04, Rice University, 1998.

[6] M. Cissé, S. Yacindag, Y. Kergosien, E. Sahin, C. Lenté, and A. Matta, "OR Problems Related to Home Health Care: A Review of Relevant Routing and Scheduling Problems," Operations Research for Health Care, vol. 13, 2017, pp. 1-22.

[7] P.M. Duque, M. Castro, K. Sörensen, and P. Goos, "Home Care Service Planning. the Case of Landelijke Thuiszorg," European Journal of Operational Research, vol. 243, 2015, pp. 292-301.

[8] C. Fikar and P. Hirsch, "Home Health Care Routing and Scheduling: A Review," Computers \& Operations Research, vol. 77, 2017, pp. 86-95.

[9] M. Gamst, and T.S. Jensen, "A branch-and-price algorithm for the long-term home care scheduling problem," Operations research proceedings, 2011, pp. 483-488.

[10] F. Grenouilleau, A. Legrain, N. Lahrichi, and L.M. Rosseau, "A Set Partitioning Heuristic for the Home Health Care Routing and Scheduling Problem," European Journal of Operational Research, vol. 275, 2019, pp. 295-303.

[11] D. S. Mankowska, F. Meisel, and C. Bierwirth, "The Home Health Care Routing and Scheduling Problem with Interdependent Services," Health Care Management Science, vol. 17, 2014, pp. 15-30.

[12] Ministry of Health, Labour and Welfare, Securing Human Resources for Longterm Care; https://www.mhlw.go.jp/file/05-Shingikai-12601000-SeisakutoukatsukanSanjikanshitsu_Shakaihoshoutantou/0000062879.pdf. at Accessed [Date 12/4/2020] in Japanese. 
[13] Ministry of Health, Labour and Welfare, Current Status of Home Medical Care; https://www.mhlw.go.jp/file/05-Shingikai-10801000-IseikyokuSoumuka/0000129546.pdf. at Accessed [Date 12/4/2020] in Japanese.

[14] Ministry of Health, Labour and Welfare, Current Status of Insured Long-term Care Service; https://www.mhlw.go.jp/toukei/list/84-1.html. at Accessed [Date 12/4/2020] in Japanese.

[15] D.C. Paraskevopoulos, G. Laporte, P.P. Repoussis, and C.D. Tarantilis, "Resource Constrained Routing and Scheduling: Review and Research Prospects," European Journal of Operational Research, vol. 263, 2017, pp. 737-754.

[16] The Japan Institute for Labour Policy and Training, Institute for Labour Policy report; https://www.jil.go.jp/institute/reports/2014/documents/0168-01.pdf. at Accessed [Date 12/4/2020] in Japanese.

[17] B. Yuan, R. Liu, and Z. Jiang, "A Branch-and-price Algorithm for the Home Health Care Scheduling and Routing Problem with Stochastic Service Times and Skill Requirements," International Journal of Production Research, vol. 53, no. 24, 2015, pp. $7450-7464$. 


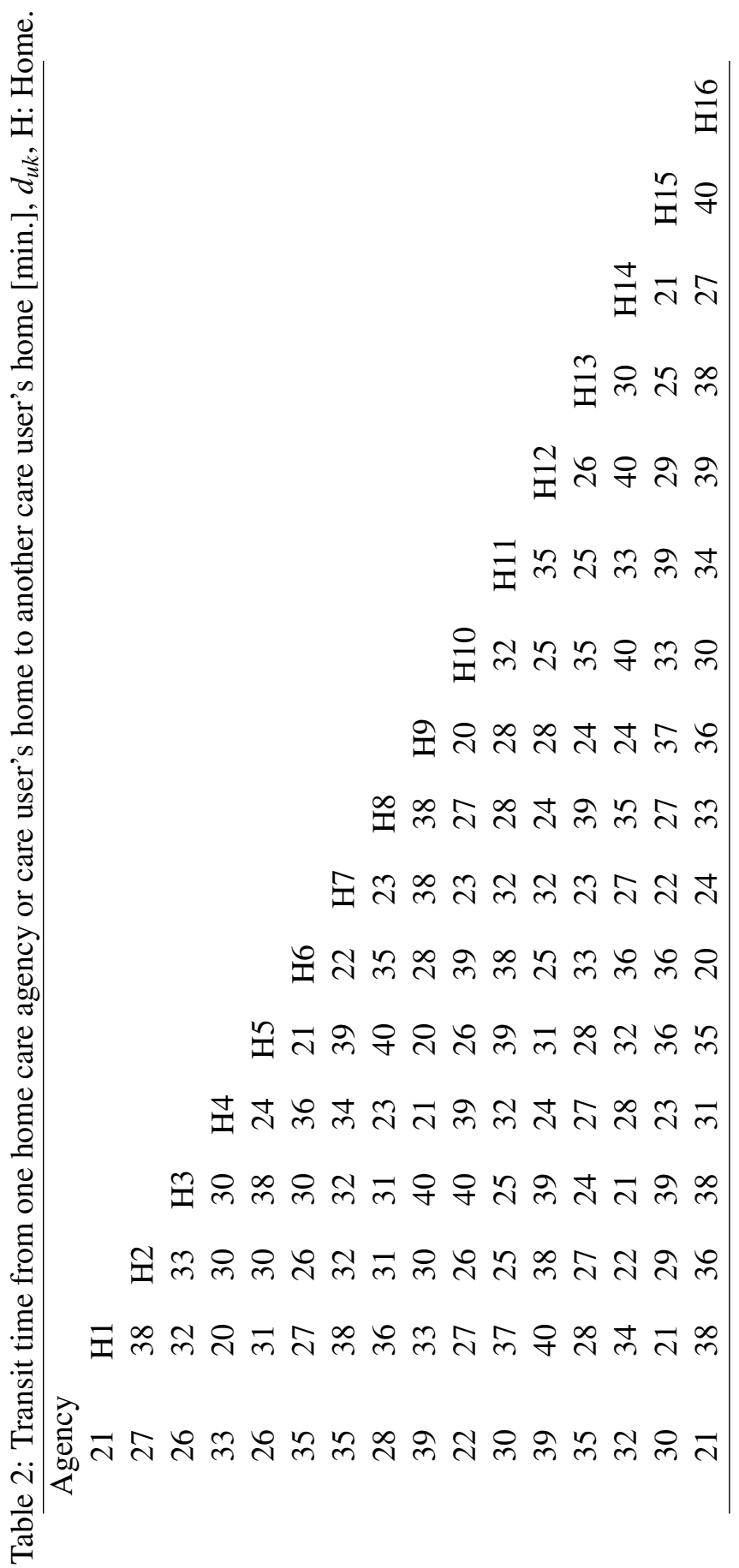




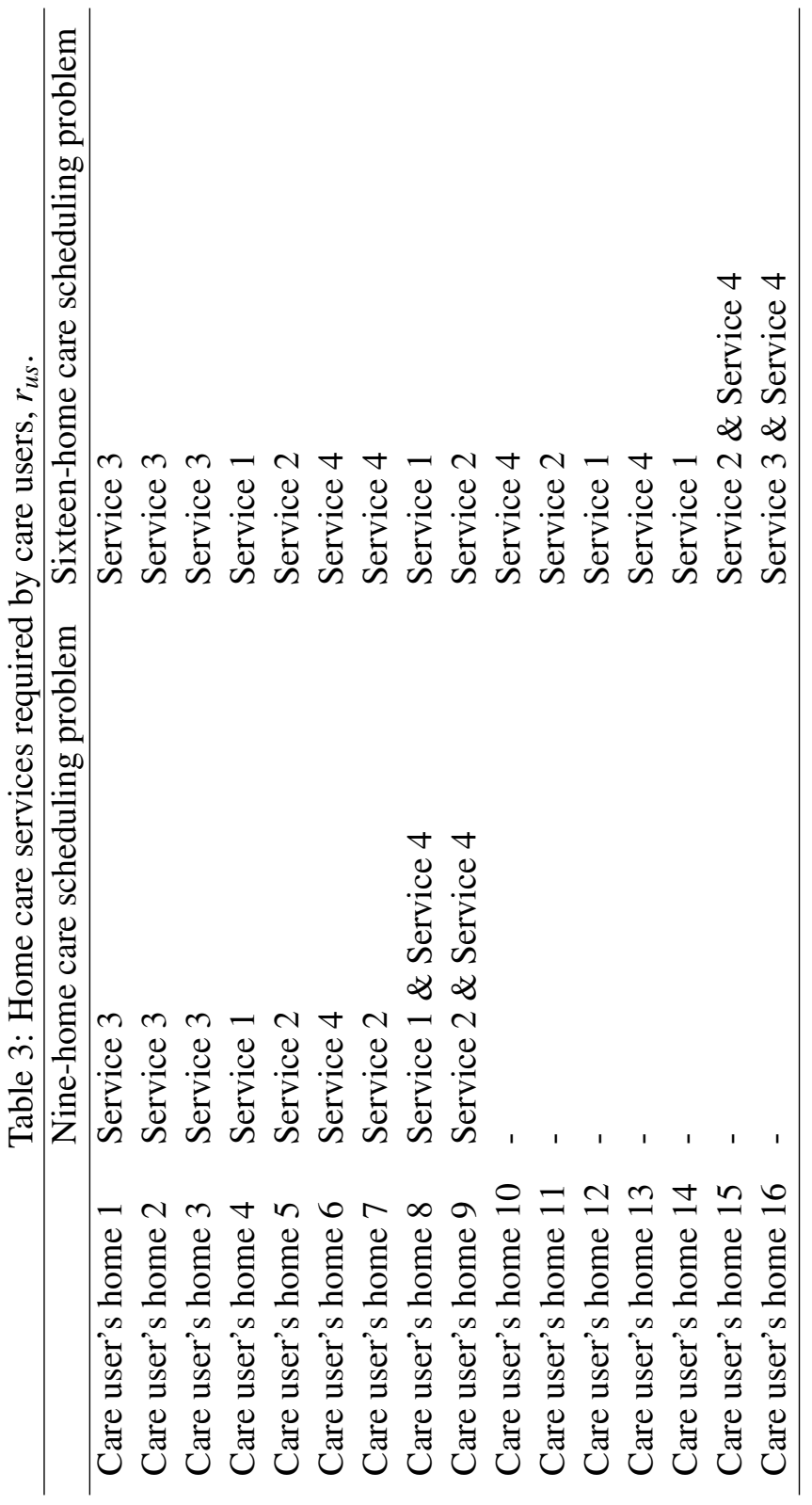




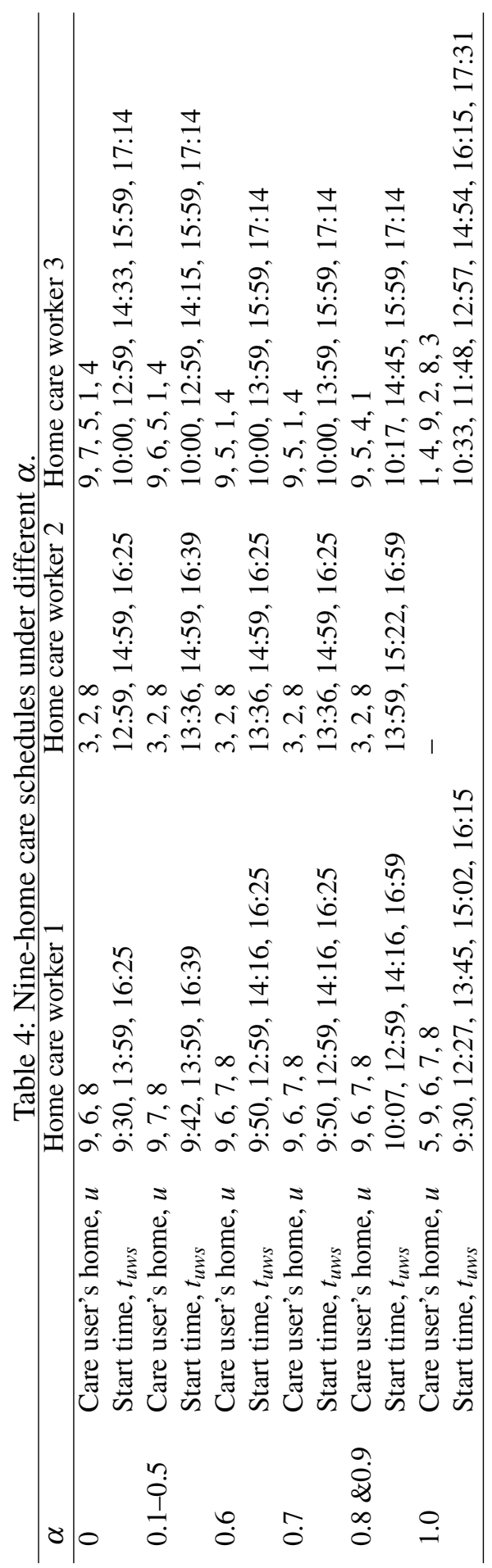


Table 5: CPU times of sixteen-home care scheduling corresponding to values of $\alpha$.

\begin{tabular}{cc}
\hline$\alpha$ & CPU time [sec.] \\
\hline 0 & 44 \\
0.1 & 49 \\
0.2 & 62 \\
0.3 & 48 \\
0.4 & 50 \\
0.5 & 95 \\
0.6 & 183 \\
0.7 & 56 \\
0.8 & 290 \\
\hline
\end{tabular}




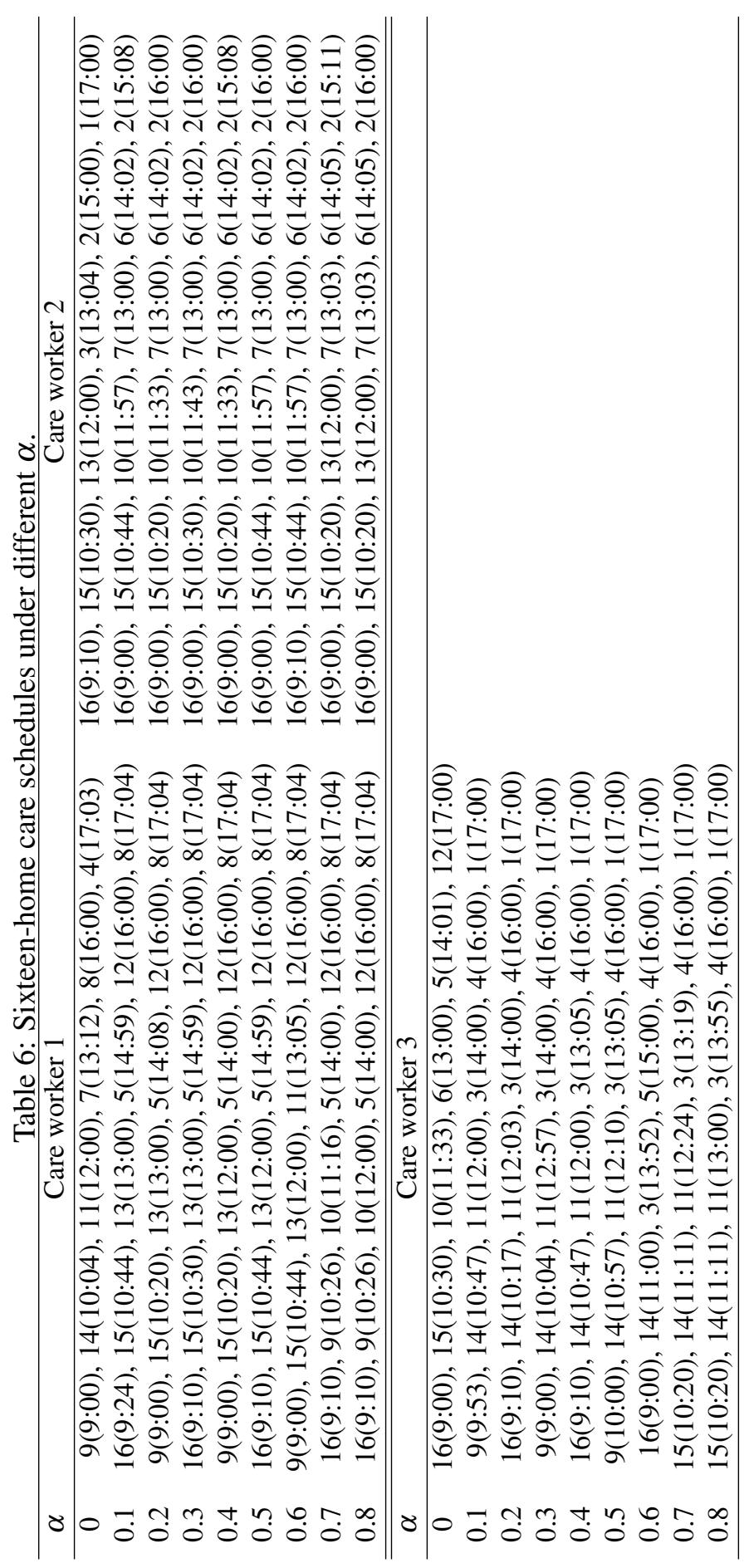

